\title{
Symptom Outcomes of Cancer Patients With Clival Metastases Treated With Radiotherapy: A Study of 44 Patients
}

\author{
REBECCA STURGIS ${ }^{1}$, ALLEDA MACK ${ }^{1}$, SEONGHO KIM ${ }^{1}$, \\ JORDAN MAIER ${ }^{2}$ and ELISABETH I. HEATH ${ }^{1}$ \\ ${ }^{1}$ Department of Oncology, Wayne State University School of Medicine, Detroit, MI, U.S.A.; \\ ${ }^{2}$ Department of Radiation Oncology, Wayne State University School of Medicine, Detroit, MI, U.S.A.
}

\begin{abstract}
Background/Aim: To describe clinical features, radiotherapy $(R T)$, and symptom outcomes in cancer patients with cranial nerve palsies associated with clival metastases. Patients and Methods: This is a retrospective review of patients with primary metastatic cancers who developed clival metastases and received $R T$ (2000-2020). Results: Of the 44 patients with primary cancers (manly breast, prostate and multiple myeloma cancers) and distal clival metastases, 32 patients (73\%) also had cervical spine metastases. Of the 23 $R T$-treated patients, $65 \%$ and $35 \%$ received clivus only and whole brain $R T$, respectively. Post-RT symptom improvement was observed in patients with diplopia $(5 / 6 ; 83 \%)$, headache (8/10; 80\%), chin numbness $(2 / 4 ; 50 \%)$, blurry vision $(2 / 5$; $40 \%)$, lateral gaze deficit $(2 / 6 ; 33 \%)$, and tongue deviation (1/4; 25\%). Conclusion: Early detection and cranial nerve examination, in addition to $R T$ treatment, should be considered in patients with breast, prostate, and multiple myeloma cancers, who developed cervical spine metastases.
\end{abstract}

Breast, prostate, kidney, lung, thyroid, and bladder cancers are cancer types that commonly spread to the bones (1). About $70 \%$ of patients with advanced breast cancer and prostate cancer have metastatic bone disease (2). Typically, metastases spread to the bones of the spine, pelvis, ribs, upper arms and thighs. Rarely, metastases can end up at the base of the skull in the clivus region. Clival tumors represent only $0.1-0.4 \%$ of all intracranial tumors, and clival metastases are an extremely rare subset of clival tumors,

This article is freely accessible online.

Correspondence to: Dr. Elisabeth I. Heath, Wayne State University Karmanos Cancer Institute, Hudson-Webber Cancer Research Center Bldg., Room 4234, 4100 John R, Detroit, MI 48201, U.S.A. Tel: +1 3135768624, Fax: +1 3135768767, e-mail: heathe@karmanos.org

Key Words: Clival metastasis, cranial nerve palsy, radiotherapy, symptom improvement. with only 57 cases reported in the literature (3-5). In the clivus, cranial nerve VI (CNVI) runs through Dorello's canal, and many patients with clival metastases present with unilateral or bilateral CNVI palsy $(4,6)$. Given that CNVI channeled by Dorello's canal reaches the neighboring cavernous sinus, through which other cranial nerves traverse, involvement of cranial nerves other than CNVI, indicates spread of clival metastasis into the cavernous sinus $(5,6)$. Because clival metastasis spreads proximal to critical structures other that cranial nerves, including basilar artery, internal carotid arteries, and brain stem, considerable morbidity and mortality burden is associated with the condition in the absence of timely treatment (5). The overall median survival of patients with clival metastases is about 2.5 years (7), and the average survival of patients with cranial nerve palsy involvement is only 5 months (7).

Clival metastases originate most frequently from prostate, kidney, and liver cancers (4). Other primary tumors from which clival metastasis can spread include breast cancer, melanoma skin, tonsillar, lung, gastrointestinal, and blood cancers $(8,9)$. Prostate cancer is the most common cancer type associated with clival metastasis, often accompanied by multiple cranial nerve deficits $(3,6)$. McDermott and coworkers (10) reported cranial nerve deficits suffered by 15 patients with metastatic prostate cancer. The presenting symptoms included facial numbness, tongue weakness, headache, diplopia, ptosis, proptosis, and unilateral blindness. All patients received external beam radiotherapy (RT) to the brain or the base of the skull. Ten of 15 patients $(67 \%)$ had complete resolution of their cranial neuropathies, and 4 patients (27\%) experienced improvement in symptoms, notwithstanding incomplete resolution of the neurologic impairments. Because of its potential for symptom improvement, RT was proposed as a treatment for metastatic prostate cancer patients with cranial nerve deficits. To contribute to the paucity of case series in the literature, we report in the present study clinical features, RT, and symptom outcomes in cancer patients with cranial nerve palsies associated with clival metastases. 


\section{Patients and Methods}

Data collection. Under the Wayne State University Institutional Review Board approval, we conducted a retrospective record review for the period in between 2000 and 2020 for patients with primary metastatic cancers, who developed distal clival metastases, and were treated with RT at the Karmanos Cancer Institute (Detroit, MI, USA). Data were collected on the patients' demographics, the primary site of a metastatic cancer, the diagnostic imaging, the type of RT, neurologic symptoms present at the time of clival diagnosis, and symptom responses to RT.

Statistical analysis. Baseline patient characteristics were summarized using count and percentage for categorical variables, and median with range for continuous variables. All statistical analyses were descriptive and did not include $p$-value calculations.

\section{Results}

Patients' characteristics. As shown in Table I, 44 patients (24 men and 20 women) were identified who met the selection criteria. The median age was 62.5 years (range $=39$ 88 years). Twenty-six patients (59\%) were white, and 16 patients $(36 \%)$ were black. Nineteen patients $(43 \%)$ had breast cancer, 18 patients (41\%) had prostate cancer, and 6 patients (14\%) had multiple myeloma. Additional cancer types included thyroid cancer, tongue squamous cell carcinoma, olfactory neuroblastoma, lung carcinoma, B-cell lymphoblastic leukemia, and diffuse large B-cell lymphoma, and were diagnosed in one patient each (2\%; not shown).

Diagnostic procedures. Imaging procedures were performed before and during diagnosis of clival metastases, including magnetic resonance imaging (MRI) of the brain in 41 patients (93\%), abdominal and thoracic computed tomography (CT) scans in 34 patients (77\%), pelvic CT in 33 patients $(75 \%)$, and whole-body bone scan in 32 patients (73\%; Table I). Of note, 32 of all 44 patients $(73 \%)$ diagnosed with clival metastases were also diagnosed with cervical spine metastases. Bone marrow biopsy of the pelvis was performed in 7 of 44 patients $(16 \%)$ as part of their clinical care, irrespective of clival metastasis diagnosis.

$R T$ treatment. Twenty-three of 44 patients (52\%) were treated with RT. Median time from diagnosis of clival metastasis to the start of RT was 9 days (range $=0-405$ days). Sixteen of 23 RT-treated patients $(70 \%)$ had cervical spine metastases. The time from the diagnosis of cervical spine metastasis to completion of RT treatment ranged from 7 to 419 days, with the median time of 23 days. As depicted in Table I, 16 of 24 men $(70 \%)$ received RT, whereas only 7 of 20 women $(30 \%)$ did. The median age of RT-treated patients was 63 years (range $=41-88$ years). Fourteen of the 23 RTtreated patients $(61 \%)$ were Whites, and 8 patients $(35 \%)$ were African Americans. Among the 23 RT-treated patients,
Table I. Patient characteristics and procedures.

\begin{tabular}{lcc}
\hline Variables & All & RT \\
& $(\mathrm{n}=44)$ & $(\mathrm{n}=23)$ \\
\hline Gender & & \\
Men & $24(55 \%)$ & $16(70 \%)$ \\
$\quad$ Women & $20(45 \%)^{*}$ & $7(30 \%)^{*}$ \\
Median age (range) & $62.5(39,88)$ & $63(41,88)$ \\
Race & & \\
White & $26(59 \%)$ & $14(61 \%)$ \\
Black & $6(36 \%)$ & $8(35 \%)$ \\
Multiple & $1(2 \%)$ & $1(4 \%)$ \\
European & $1(2 \%)$ & $0(0 \%)$ \\
Breast cancer & & \\
No & $25(57 \%)$ & $17(74 \%)$ \\
Yes & $19(43 \%)$ & $6(26 \%)$ \\
Prostate cancer & & \\
No & $26(59 \%)$ & $13(57 \%)$ \\
Yes & $18(41 \%)$ & $10(43 \%)$ \\
Multiple myeloma & & \\
No & $38(86 \%)$ & $19(83 \%)$ \\
Yes & $6(14 \%)$ & $4(17 \%)$ \\
Imaging \\
MRI of the brain
\end{tabular}

CT: Computed tomography; RT: radiotherapy. *Percentage was calculated for the ratio of the number of all female patients and the number of all patients in the study $(\mathrm{n}=44)$. **Percentage was calculated for the ratio of the number of female patients who were treated with RT and the number of patients who received RT $(n=23)$. Similar series of calculations were performed for the other data in the corresponding rows and columns.

6 patients (26\%) were with breast cancer, 10 patients (43\%) with prostate cancer, and 4 patients $(17 \%)$ with multiple myeloma. Twenty of the 23 RT-treated patients (87\%) received MRI of the brain, 15 patients $(65 \%)$ got either abdominal CT or thoracic CT scan, or whole-body bone scan, and 14 patients $(61 \%)$ received pelvic CT. Bone marrow biopsy of the pelvis was performed in five of the 23 RT-treated patients (22\%; not shown).

Fifteen of the 23 RT-treated patients (65\%) received clivus only RT. Eight patients (35\%), who besides clival metastases 
Table II. Patients not treated with RT or with unknown RT status.

\begin{tabular}{lcc}
\hline Cancer type & $\begin{array}{c}\text { No RT } \\
(\mathrm{n}=7)\end{array}$ & $\begin{array}{c}\text { Unknown RT status } \\
(\mathrm{n}=14)\end{array}$ \\
\hline Prostate cancer & $5(11 \%)$ & $2(5 \%)$ \\
Breast cancer & $1(2 \%)$ & $11(25 \%)$ \\
Breast cancer/MM & $1(2 \%)$ & - \\
Prostate cancer/MM & - & $1(2 \%)$ \\
\hline
\end{tabular}

MM: Multiple myeloma; RT: radiotherapy. Percentage was calculated for the ratio of the number of patients with cancer and the number of all the patients in the study $(n=44)$

also developed brain metastases, were treated with whole brain RT. Of all 15 patients who were treated with clivus only RT, 2 patients (13.3\%) also received RT to the cervical spine (not shown). Among clivus only RT-treated patients, 12 patients received 30 Grays (Gy; in $3 \mathrm{~Gy} \times 10$ fractions), one patient received a total of $20 \mathrm{~Gy}$ (in $4 \mathrm{~Gy} \times 5$ fractions), and one patient was given an $8 \mathrm{~Gy} \times 1$ treatment only. One patient, previously treated in the base of the skull region, received a lower dose of $2.5 \mathrm{~Gy} \times 10$ fractions using intensity-modulated RT to minimize overlap with the previous treatment. Among whole brain RT-treated patients, 6 patients were given 30 Gy in 10 fractions, one patient received 25 Gy in 10 fractions, and one patient was treated with a total of $12 \mathrm{~Gy}(3 \mathrm{~Gy} \times 4)$ out of $30 \mathrm{~Gy}(3 \mathrm{~Gy} \times 10)$ planned treatment prior to discontinuing due to worsening of the performance status.

Of all 44 patients in the study, 7 patients (15.9\%) expired within 3 months after the diagnosis of clival metastasis, and ended up receiving no RT treatment (Table II). Among them, 5 patients $(11.4 \%)$ were with prostate cancer, one patient $(2.3 \%)$ was with breast cancer, and one patient $(2.3 \%)$ was diagnosed with both breast cancer and multiple myeloma. Of note, 14 patients $(31.8 \%)$, who received care outside our institution, were with unknown RT status. Among them, 2 patients $(4.5 \%)$ were with prostate cancer, 11 patients (25.0\%) were diagnosed with breast cancer, and one patient $(2.3 \%)$ was with both prostate cancer and multiple myeloma.

Types of neurologic symptoms. Common neurologic symptoms noted in all 44 patients are depicted in Table III. Thirteen patients $(30 \%)$ suffered from headache, 7 patients $(16 \%)$ experienced diplopia, 6 patients $(14 \%)$ had lateral gaze paralysis, 5 patients $(11 \%)$ presented with blurry vision or chin numbness, and 4 patients (9\%) suffered from tongue deviation. Some patients experienced more than one symptom.

Table III also shows neurologic symptoms based on cancer type. Patients with breast cancer experienced headache $(6 / 13 ; 46 \%)$, and blurry vision or chin numbness $(1 / 5 ; 20 \%$ each). Patients with prostate cancer had diplopia $(4 / 7 ; 57 \%)$, tongue deviation $(2 / 4 ; 50 \%)$, blurry vision or
Table III. Neurologic symptoms based on cancer type.

\begin{tabular}{lc}
\hline Cancer type & Patients \\
\hline Headache & \\
Yes & $13(30 \%)^{*}$ \\
Breast cancer & $6(46 \%)^{* *}$ \\
Prostate cancer & $3(23 \%)$ \\
Multiple myeloma & $3(23 \%)$ \\
Diplopia & \\
Yes & $7(16 \%)$ \\
Breast cancer & - \\
Prostate cancer & $4(57 \%)$ \\
Multiple myeloma & $2(29 \%)$ \\
Lateral gaze deficit & \\
Yes & $6(14 \%)$ \\
Breast cancer & - \\
Prostate cancer & $2(33 \%)$ \\
Multiple myeloma & $2(33 \%)$ \\
Blurry vision & \\
Yes & $5(11 \%)$ \\
Breast cancer & $1(20 \%)$ \\
Prostate cancer & $2(40 \%)$ \\
Multiple myeloma & $1(20 \%)$ \\
Chin numbness & \\
Yes & $5(11 \%)$ \\
Breast cancer & $1(20 \%)$ \\
Prostate cancer & $2(40 \%)$ \\
Multiple myeloma & $1(20 \%)$ \\
Tongue deviation & $4(9 \%)$ \\
Yes & - \\
Breast cancer & $2(50 \%)$ \\
Prostate cancer & $2(50 \%)$ \\
Multiple myeloma & \\
\hline & \\
\hline &
\end{tabular}

*Percentage was calculated for the ratio of the number of all patients with headache and the number of all patients in the study $(n=44)$. Similar calculations were performed for patients with other symptoms. **Percentage was calculated for the ratio of the number of breast cancer patients with headache and the number of all patients with headache. Similar calculations were performed for patients with other cancer types for each symptom.

chin numbness $(2 / 5 ; 40 \%$ each), lateral gaze deficit $(2 / 6$; $33 \%)$, and headache $(3 / 13 ; 23 \%)$. The symptoms noted in patients with multiple myeloma were tongue deviation $(2 / 4$; $50 \%)$, lateral gaze deficit $(2 / 6 ; 33 \%)$, diplopia $(2 / 7 ; 29 \%)$, and headache $(3 / 13 ; 23 \%)$, in addition to blurry vision or chin numbness $(1 / 5 ; 20 \%$ each).

Symptom improvement after RT. Symptom improvement after RT was defined as lessening or resolution of symptoms based on patients' reports. As shown in Table IV, symptom improvement after RT was experienced by 5 of 6 patients $(83 \%)$ with diplopia, 8 of 10 patients $(80 \%)$ with headache, 2 of 4 patients $(50 \%)$ with chin numbness, 2 of 5 patients $(40 \%)$ with blurry vision, 2 of 6 patients (33\%) with lateral gaze deficit, and one of 4 patients (25\%) with tongue deviation. 
Table IV. Neurologic symptoms before and after RT.

\begin{tabular}{lcc}
\hline Symptom & Present before RT & Improved after RT \\
\hline Headache & $10(43 \%)^{*}$ & $8(80 \%)^{* *}$ \\
Diplopia & $6(26 \%)$ & $5(83 \%)$ \\
Lateral gaze deficit & $6(26 \%)$ & $2(33 \%)$ \\
Blurry vision & $5(22 \%)$ & $2(40 \%)$ \\
Chin numbness & $4(17 \%)$ & $2(50 \%)$ \\
Tongue deviation & $4(17 \%)$ & $1(25 \%)$ \\
\hline
\end{tabular}

RT: Radiotherapy. *Percentage was calculated for the ratio of the number of patients with a headache before RT and the number of all RT-treated patients $(n=23)$. **Percentage was calculated for the ratio of the number of patients who experienced lessening or resolution of their headache post-RT and the number of all patients with the headache before RT. Similar calculations were performed for patients with other symptoms in the corresponding rows and columns.

A classic example of the patients in this study is a 58-yearold patient with metastatic castrate-resistant prostate cancer, who initially presented with severe headaches. Figure 1 shows that the clival involvement was noted using the MRI scan. In this case, the RT treatment plan was 3,000 centigrays (cGy) delivered in 10 fractions using opposed laterals and 6 megavolts (MV) photons. Figure 2 shows that the total dose of 3,000 cGy was delivered to the entire gross tumor volume, and to $95 \%$ of the planning target volume. The RT treatment resulted in significant improvement of headaches.

\section{Discussion}

Although $70 \%$ of patients with advanced breast cancer and prostate cancer have metastatic bone disease (2), the presence of clival metastases is not common (3-5). When clival metastases are present, however, the resulting neurologic symptoms, such as facial numbness, visual changes, and tongue paralysis, can be devastating. Dramatic clinical findings associated with clival pathology are known to be nonspecific, and the time interval from the diagnosis of the primary tumor to the diagnosis of the clival metastasis has a wide range from 2 months to 33 years (4, 11). In this study, ascertaining the time interval from the onset of neurologic symptoms to the diagnosis of clival metastasis by brain MRI was a challenge. In addition, the diagnosis of clival metastasis was delayed, possibly due to the heterogeneous nature of the cranial neuropathies and symptoms.

The RT-treated patients in this study experienced symptom improvement with variable responses. Patients with headache and diplopia improved after RT. However, patients with blurry vision, eye gaze paralysis, and tongue deviation are not guaranteed any improvement. This may be due to the rapid progression of the disease, which is consistent with the observation that 7 patients who did not receive RT (16\%; 7/44) died within 3 months after the diagnosis of clival

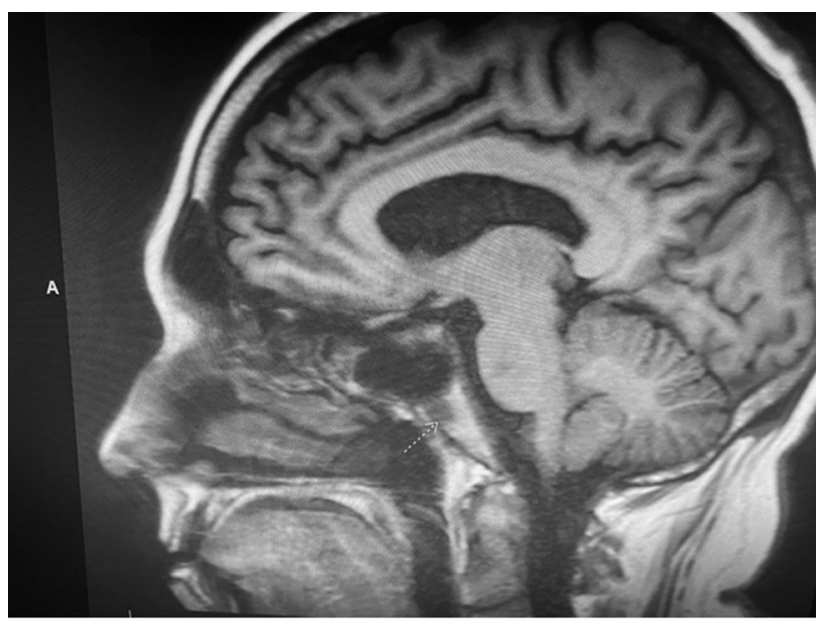

Figure 1. Brain magnetic resonance imaging (sagittal view, with an arrow pointing to the clivus) of a 58-year-old patient with prostate cancer (stage 4).

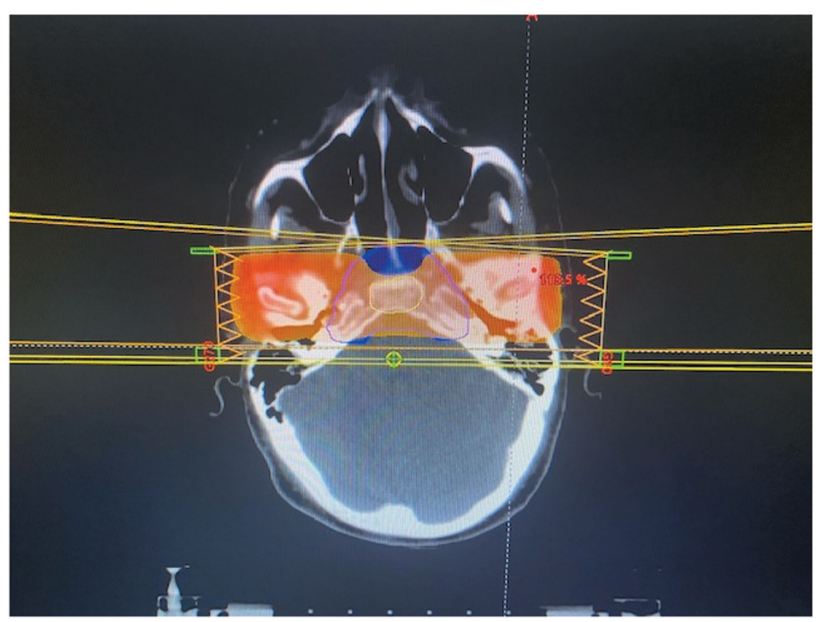

Figure 2. Radiation treatment plan (axial view showing GTV (yellow) and PTV (blue). The total dose (3,000 cGy) was delivered to the entire GTV, and to $95 \%$ of the PTV. cGy: Centigray; GTV: gross tumor volume; $M V$ : megavolts; PTV: planning target volume.

metastasis. The development of clival metastasis typically occurs later in the patient's disease continuum. However, when the condition is diagnosed early, and the RT treatment is planned, the median time from the diagnosis to the start of RT is 9 days. Early recognition and treatment of clival metastasis may provide improved symptom relief.

Among 23 patients who received RT, 16 patients (70\%) were men and 7 patients $(30 \%)$ were women. Furthermore, of all 23 RT-treated patients, 14 patients $(61 \%)$ were whites and 8 patients $(35 \%)$ were blacks. The data suggest trends in 
gender and racial disparities. A literature review found a preponderance of men who were reported with clival metastases $(72 \%, 31 / 43)$ (11). In our study, however, the gender disparity may be apparent, because 11 women with breast cancer $(25 \%$; 11/44) received care outside our institution, and possibly were treated with RT elsewhere.

Understanding the pathophysiology of clival metastasis may be improved by our knowledge of chordoma, a rare type of sarcoma that develops de novo from the base of the skull. The most prominent single nucleotide polymorphism rs2305089 in the $\mathrm{T}$ (brachyury) gene has been associated with a 6-fold increase in the risk of developing chordoma, and has been implicated in the prognosis of individuals with chordoma (12, 13). Genomic profiles of 27 clivus chordoma patients derived from the biopsy samples were reported in (14). These patients were among the youngest in the chordoma patient group, with the median age of 43 years, and had genomic alterations in $C D K N 2 A, C D K N 2 B, P B R M 1$, and PTCH1. In our study, however, these genomic changes were not observed in the genomic profiling data obtained from a small subgroup of patients (not shown). Consistent with an important role of genomic sequencing in the management of solid tumor cancer patients, future evaluation of genomic signatures associated with clival metastases is warranted.

One of the strengths of our study is showing symptomatic improvement of cranial neuropathies after radiation therapy. Furthermore, long term side effects, such as radiation necrosis, were not experienced by the patients. Because the clivus is a rare site of bony spread of metastatic disease, our study contributes to the paucity of case series in the literature. In addition, these findings may have an impact on counseling patients with clival metastasis by treating physicians. Limitations of the present report include its retrospective and monocentric nature, in addition to small sample size, and missing data for patients who received care outside our institution and whose RT status was unknown.

\section{Conclusion}

Our data suggest that (i) early detection and incorporation of detailed cranial nerve examination are needed, especially in patients with breast cancer, prostate cancer, and multiple myeloma, who developed metastases in the cervical spine, and that (ii) palliative RT should be considered in these patients, given its potential for symptom improvement. Our data imply that providers need to recognize a potential link between neurologic deficits and metastatic carcinomas, in order to effectively diagnose and treat the patients, with the goal of symptom improvement. This is consistent with the findings that treatment of neurologic defects is associated with improvement of patients' emotional wellbeing and quality of life, and that psychological wellness and positive health outcomes are related $(15,16)$.

\section{Conflicts of Interest}

None of the Authors have any conflicts of interest to declare regarding this study.

\section{Authors' Contributions}

RS contributed to the data acquisition and analysis, and writing up the manuscript. AM contributed to the data acquisition. SK and JM contributed to the study's design, and played a major role in data acquisition, analysis and interpretation, as well as writing up the manuscript. EIH designed the study, revised data acquisition, analysis and interpretation, and was primarily responsible for writing the manuscript. All Authors approved the submission.

\section{Acknowledgements}

The work described here was presented at the Michigan American College of Physicians Annual Fall Virtual Scientific Meeting on October 18, 2020.

\section{References}

1 Schmid-Alliana A, Schmid-Antomarchi H, Al-Sahlanee R, Lagadec P, Scimeca JC and Verron E: Understanding the progression of bone metastases to identify novel therapeutic targets. Int J Mol Sci 19(1): 148, 2018. PMID: 29300334. DOI: 10.3390/ijms 19010148

2 Buijs JT and van der Pluijm G: Osteotropic cancers: from primary tumor to bone. Cancer Lett 273(2): 177-193, 2009. PMID: 18632203. DOI: 10.1016/j.canlet.2008.05.044

3 Pallini R, Sabatino G, Doglietto F, Lauretti L, Fernandez E and Maira G: Clivus metastases: report of seven patients and literature review. Acta Neurochir (Wien) 151(4): 291-6; discussion 296, 2009. PMID: 19259614. DOI: 10.1007/s00701009-0229-1

4 Dekker SE, Wasman J, Yoo KK, Alonso F, Tarr RW, Bambakidis NC and Rodriguez K: Clival metastasis of a duodenal adenocarcinoma: a case report and literature review. World Neurosurg 100: 62-68, 2017. PMID: 28034818. DOI: 10.1016/ j.wneu.2016.12.078

5 Cathel A, Khan YR, Blais D, Mahato B and Mahato D: Metastatic disease to clivus: Biopsy or not? Cureus 11(9): e5658, 2019. PMID: 31720134. DOI: $10.7759 /$ cureus.5658

6 Lodhia $\mathrm{V}$ and Puspanathan T: Insidious enemy: downside to prolonged survival in prostate cancer. BMJ Case Rep 2017: bcr2017220613, 2017. PMID: 28768673. DOI: 10.1136/bcr2017-220613

7 Marchese-Ragona R, Maria Ferraro S, Marioni G, Staffieri C, Manara R, Restivo DA and Staffieri A: Abducent nerve paralysis: first clinical sign of clivus metastasis from tonsillar carcinoma. Acta Otolaryngol 128(6): 713-716, 2008. PMID: 18568510. DOI: $10.1080 / 00016480701687283$

8 Okudo J and Anusim N: Unusual spread of renal cell carcinoma to the clivus with cranial nerve deficit. Case Rep Neurol Med 2016: 9184501, 2016. PMID: 27110412. DOI: 10.1155/2016/9184501

9 García-Álvarez SM, Olondo-Zulueta L, Pericás JM, Colomo L and Bosch X: Numb chin syndrome with vagal and hypoglossal paralysis: an initial sign of an uncommon diagnosis. Am J Med 
Sci 344(3): 241-244, 2012. PMID: 22739563. DOI: 10.1097/ MAJ.0b013e318256050f

10 McDermott RS, Anderson PR, Greenberg RE, Milestone BN and Hudes GR: Cranial nerve deficits in patients with metastatic prostate carcinoma: clinical features and treatment outcomes. Cancer 101(7): 1639-1643, 2004. PMID: 15468187. DOI: $10.1002 /$ cncr.20553

11 Deconde AS, Sanaiha Y, Suh JD, Bhuta S, Bergsneider M and Wang MB: Metastatic disease to the clivus mimicking clival chordomas. J Neurol Surg B Skull Base 74(5): 292-299, 2013. PMID: 24436927. DOI: 10.1055/s-0033-1348027

12 Pillay N, Plagnol V, Tarpey PS, Lobo SB, Presneau N, Szuhai K, Halai D, Berisha F, Cannon SR, Mead S, Kasperaviciute D, Palmen J, Talmud PJ, Kindblom LG, Amary MF, Tirabosco R and Flanagan AM: A common single-nucleotide variant in $\mathrm{T}$ is strongly associated with chordoma. Nat Genet 44(11): 11851187, 2012. PMID: 23064415. DOI: $10.1038 / \mathrm{ng} .2419$

13 Bettegowda C, Yip S, Lo SL, Fisher CG, Boriani S, Rhines LD, Wang JY, Lazary A, Gambarotti M, Wang WL, Luzzati A, Dekutoski MB, Bilsky MH, Chou D, Fehlings MG, McCarthy EF, Quraishi NA, Reynolds JJ, Sciubba DM, Williams RP, Wolinsky JP, Zadnik PL, Zhang M, Germscheid NM, Kalampoki V, Varga PP, Gokaslan ZL and AOSpine Knowledge Forum Tumor: Spinal column chordoma: prognostic significance of clinical variables and T (brachyury) gene SNP rs2305089 for local recurrence and overall survival. Neuro Oncol 19(3): 405413, 2017. PMID: 27663388. DOI: 10.1093/neuonc/now 156
14 Killian J, Yang X, Danziger N, Elvin J, Vergilio J, Lin D, Williams E, Ramkissoon S, Severson E, Hemmerich A, Duncan D, Edgerly C, Huang R, Hiemenz M, Reddy V, Mcgregor K, Venstrom J, Schrock A, Alexander B and Ross J: Differential genomic landscape of clinically advanced/metastatic chordomas (mChor) based on primary tumor site. Journal of Clinical Oncology 38(15_suppl): 11521-11521, 2020. DOI: 10.1200/JCO.2020.38.15_suppl.11521

15 Quan GM, Vital JM, Aurouer N, Obeid I, Palussière J, Diallo A and Pointillart V: Surgery improves pain, function and quality of life in patients with spinal metastases: a prospective study on 118 patients. Eur Spine J 20(11): 1970-1978, 2011. PMID: 21706361. DOI: 10.1007/s00586-011-1867-6

16 Salsman JM, Victorson D, Choi SW, Peterman AH, Heinemann AW, Nowinski C and Cella D: Development and validation of the positive affect and well-being scale for the neurology quality of life (Neuro-QOL) measurement system. Qual Life Res 22(9): 2569-2580, 2013. PMID: 23526093. DOI: 10.1007/s11136-0130382-0
Received July 23, 2021

Revised August 24, 2021

Accepted August 25, 2021 